\title{
Growth of solutions of some kinds of linear difference equations
}

\author{
Shun-Zhou Wu and Xiu-Min Zheng*
}

"Correspondence:

zhengxiumin2008@sina.com

Institute of Mathematics and Information Science, Jiangxi Normal

University, Nanchang, Jiangxi

330022, P.R. China

\begin{abstract}
In this paper, we investigate the growth and value distribution of solutions of some kinds of linear difference equations, where there may be more than one coefficient having the same maximal order and the same maximal type.
\end{abstract}

MSC: $30 \mathrm{D} 35 ; 39 \mathrm{~A} 22$

Keywords: linear difference equation; meromorphic solution; growth; exponent of convergence

\section{Introduction}

Throughout this paper, we use the standard notations and basic results of Nevanlinna's value distribution theory (see [1-4]). In addition, we use the notations $\sigma(f), \sigma_{2}(f)$ and $\lambda(f-a)$ to denote, respectively, the order, the hyperorder, and the exponent of convergence of the sequence of $a$-points of a meromorphic function $f(z)$ in the complex plane, where $a \in \mathbb{C} \cup\{\infty\}$. Furthermore, we can get the definition of $\lambda(f-\varphi)$, when $a$ is replaced by a meromorphic function $\varphi(z)$.

Recently, with the research and further development of difference analogs of Nevanlinna's theory, it has been applied more and more widely in the difference field. By this important tool, many scholars investigated the linear difference equation

$$
A_{k}(z) f\left(z+c_{k}\right)+\cdots+A_{1}(z) f\left(z+c_{1}\right)+A_{0}(z) f(z)=0,
$$

where $k \in \mathbb{N}_{+}$and $c_{j}, j=1, \ldots, k$ are distinct nonzero complex constants, and obtained many results on the growth and the exponent of convergence of the sequence of zeros of meromorphic solutions of (1.1). For instance, in [5-7], the authors considered the case when there is exactly only one coefficient of (1.1) having the maximal order; in [8-10], the authors considered the case when there is exactly only one coefficient having the type strictly greater than the others among those having the maximal order; and in $[5,6,11]$, the authors considered the case when the coefficients of (1.1) are polynomials.

Further, how about the case when there are more than one coefficient having the same maximal order and the same maximal type?

In 2013, Liu [12] considered the growth and the exponent of convergence of the sequence of small function value points of second order linear difference equations and obtained the following theorem.

\section{照 Springer}


Theorem A (see [12]) Let $c_{1}, c_{2}\left(\neq c_{1}\right)$, a be nonzero constants, $h_{1}(z)$ be a nonzero meromorphic functions with $\sigma\left(h_{1}\right)<1, B(z)$ be a nonzero meromorphic function. If $B(z)$ satisfies any one of the following three conditions:

(i) $\sigma(B)>1$ and $\delta(\infty, B)>0$;

(ii) $\sigma(B)<1$;

(iii) $B(z)=h_{0}(z) e^{b z}$, where $b$ is a nonzero constant, $h_{0}(z)(\not \equiv)$ is a meromorphic function with $\sigma\left(h_{0}\right)<1$,

then every meromorphic solution $f(z)(\not \equiv)$ of the difference equation

$$
f\left(z+c_{2}\right)+h_{1}(z) e^{a z} f\left(z+c_{1}\right)+B(z) f(z)=0
$$

satisfies $\sigma(f) \geq \max \{\sigma(B), 1\}+1$. Further, if $\varphi(z)(\not \equiv 0)$ is a meromorphic function with $\sigma(\varphi)<\max \{\sigma(B), 1\}+1$, then $\lambda(f-\varphi)=\sigma(f) \geq \max \{\sigma(B), 1\}+1$.

Liu and Mao [13] considered the growth of meromorphic solutions of (1.1), where the coefficients may have the same order and the same type, and obtained the following theorem.

Theorem B (see [13]) Let $A_{j}(z)=h_{j}(z) e^{P_{j}(z)}+D_{j}(z), j=0,1, \ldots, k$, where $P_{j}(z)=a_{j n} z^{n}+\cdots+$ $a_{j 0}$ are polynomials with degree $n(\geq 1), h_{j}(z)(\not \equiv 0), D_{j}(z)$ are entire functions with order less than $n$. If $a_{j n}, j=0,1, \ldots, k$ are distinct complex numbers, then every meromorphic solution $f(z)(\not \equiv 0)$ of $(1.1)$ satisfies $\sigma(f) \geq \max _{0 \leq j \leq k}\left\{\sigma\left(A_{j}\right)\right\}+1$.

In this paper, we are concerned with the more general problem than Theorems A and B, and obtain the following results, which extend and improve the previous results.

First, we consider the difference equation (1.1).

Theorem 1.1 Let $k, n \in \mathbb{N}_{+}, A_{j}(z)=B_{j}(z) e^{P_{j}(z)}+D_{j}(z) e^{Q_{j}(z)}+R_{j}(z), j=0,1, \ldots, k$, where $P_{j}(z)=a_{j n} z^{n}+\cdots+a_{j 0}, Q_{j}(z)=b_{j n} z^{n}+\cdots+b_{j 0}, j=0,1, \ldots, k$ are polynomials with degree $n$ and satisfy $\left|a_{j n}\right| \geq\left|b_{j n}\right|>0, j=0,1, \ldots, k, B_{j}(z), D_{j}(z), R_{j}(z), j=0,1, \ldots, k$ are meromorphic functions and satisfy $\max _{0 \leq j \leq k}\left\{\sigma\left(B_{j}\right), \sigma\left(D_{j}\right), \sigma\left(R_{j}\right)\right\}=\omega<n, A_{j}(z)-R_{j}(z) \not \equiv 0, j=0,1, \ldots, k$. Let $c_{j}, j=1, \ldots, k$ be distinct nonzero complex constants. If there exists an $i \in\{0,1, \ldots, k\}$ such that for all $j(\neq i),\left|a_{i n}\right| \geq\left|a_{j n}\right|$, and

(i) $\arg a_{i n} \neq \arg a_{j n}$, or $\arg a_{i n}=\arg a_{j n},\left|a_{i n}\right|>\left|a_{j n}\right|$ and

(ii) $\arg a_{i n} \neq \arg b_{j n}$, or $\arg a_{i n}=\arg b_{j n},\left|a_{i n}\right|>\left|b_{j n}\right|$ hold simultaneously, then every meromorphic solution $f(z)(\not \equiv 0)$ of $(1.1)$ satisfies $\sigma(f) \geq$ $n+1$. Further, if $\varphi(z)(\not \equiv 0)$ is a meromorphic function with $\sigma(\varphi)<n+1$, then for every meromorphic solution $f(z)(\not \equiv 0)$ of $(1.1)$ with $\sigma_{2}(f)<1$, we have $\lambda(f-\varphi)=\sigma(f) \geq n+1$.

Remark 1.1 In Theorem 1.1, if there exist some $j(\neq i)$ such that $A_{j}(z)-R_{j}(z) \equiv 0$, or some of $B_{j}(z)(j \neq i), D_{j}(z), R_{j}(z), j=0,1, \ldots, k$, are equal to zero, then the corresponding result holds by using a similar proof to the one of Theorem 1.1.

Next, we consider difference operators instead of shift operators in (1.1).

For a nonzero complex constant $c$, the forward differences $\Delta^{k} f(z), k \in \mathbb{N}_{+}$, are defined (see [14]) by

$$
\Delta f(z)=\Delta^{1} f(z)=f(z+c)-f(z), \quad \Delta^{k+1} f(z)=\Delta^{k} f(z+c)-\Delta^{k} f(z), \quad k \in \mathbb{N}_{+} .
$$


It is shown in [6] that

$$
\Delta^{k} f(z)=\sum_{j=0}^{k} C_{k}^{j}(-1)^{k-j} f(z+j c), \quad f(z+k c)=\sum_{j=0}^{k} C_{k}^{j} \Delta^{j} f(z), \quad k \in \mathbb{N}_{+} .
$$

Then we can obtain the following theorem.

Theorem 1.2 Suppose that $A_{j}(z), j=0,1, \ldots, k$, satisfy the hypotheses of Theorem 1.1, and $i$ is also defined as in Theorem 1.1. If $i=0$, then every meromorphic solution $f(z)(\not \equiv)$ of the difference equation

$$
A_{k}(z) \Delta^{k} f(z)+\cdots+A_{1}(z) \Delta f(z)+A_{0}(z) f(z)=0
$$

satisfies $\sigma(f) \geq n+1$. Further, if $\varphi(z)(\not \equiv 0)$ is a meromorphic function with $\sigma(\varphi)<n+1$, then for every meromorphic solution $f(z)(\not \equiv 0)$ of $(1.2)$ with $\sigma_{2}(f)<1$, we have $\lambda(f-\varphi)=$ $\sigma(f) \geq n+1$.

In the end, we can easily get the following corollary.

Corollary 1.1 Let $a_{j} \in \mathbb{C}, j=0,1, \ldots, k$, such that $a_{0} \neq a_{j},\left|a_{0}\right| \geq\left|a_{j}\right| \geq 0, j=1, \ldots, k$, and $h_{j}(z)(\not \equiv 0), j=0,1, \ldots, k$, be meromorphic functions with order less than $n$, then every meromorphic solution $f(z)(\not \equiv 0)$ of the difference equation

$$
\begin{aligned}
& h_{k}(z) e^{a_{k} z^{n}} \Delta^{k} f(z)+h_{k-1}(z) e^{a_{k-1} z^{n}} \Delta^{k-1} f(z)+\cdots+h_{1}(z) e^{a_{1} z^{n}} \Delta f(z) \\
& \quad+h_{0}(z) e^{a_{0} z^{n}} f(z)=0
\end{aligned}
$$

satisfies $\sigma(f) \geq n+1$. Further, if $\varphi(z)(\not \equiv 0)$ is a meromorphic function with $\sigma(\varphi)<n+1$, then for every meromorphic solution $f(z)(\not \equiv 0)$ with $\sigma_{2}(f)<1$, we have $\lambda(f-\varphi)=\sigma(f) \geq n+1$.

\section{Preliminary lemmas}

Lemma 2.1 (see [6]) Let $\eta_{1}, \eta_{2}$ be two arbitrary complex numbers, and $f(z)$ be a meromorphic function of finite order $\sigma$. Let $\varepsilon>0$ be given, then there exists a set $E \subset(0,+\infty)$ with finite logarithmic measure such that for all $r \notin E \cup[0,1]$, we have

$$
\exp \left(-r^{\sigma-1+\varepsilon}\right) \leq\left|\frac{f\left(z+\eta_{1}\right)}{f\left(z+\eta_{2}\right)}\right| \leq \exp \left(r^{\sigma-1+\varepsilon}\right)
$$

Remark 2.1 It follows from Lemma 2.1 that

$$
\begin{aligned}
\left|\frac{\Delta^{i} f(z)}{f(z)}\right| & =\left|\frac{\sum_{j=0}^{i} C_{i}^{j}(-1)^{i-j} f(z+j c)}{f(z)}\right| \leq \sum_{j=0}^{i} C_{i}^{j}\left|\frac{f(z+j c)}{f(z)}\right| \\
& \leq \sum_{j=0}^{i} C_{i}^{j} \exp \left\{r^{\sigma-1+\varepsilon}\right\}=2^{i} \exp \left\{r^{\sigma-1+\varepsilon}\right\}, \quad i \in \mathbb{N}_{+} .
\end{aligned}
$$

Lemma 2.2 (see [15]) Let $f(z)$ be a meromorphic function with $\sigma(f)=\beta<+\infty$, then for any given $\varepsilon>0$, there exists a set $E \subset[0,+\infty)$ with $m E<+\infty$ such that for all $z$ with $|z|=$ 
$r \notin[0,1] \cup E, r \rightarrow \infty$, we have

$$
\exp \left\{-r^{\beta+\varepsilon}\right\} \leq|f(z)| \leq \exp \left\{r^{\beta+\varepsilon}\right\}
$$

Lemma 2.3 (see [16]) Suppose that $P(z)=(\alpha+\beta i) z^{n}+\cdots(\alpha, \beta$ are real numbers such that $|\alpha|+|\beta| \neq 0)$ is a polynomial with degree $n(\geq 1)$, and $\omega(z)(\not \equiv 0)$ is a meromorphic function with $\sigma(\omega)<n$. Set $g(z)=\omega(z) e^{P(z)}, z=r e^{i \theta}, \delta(P, \theta)=\alpha \cos n \theta-\beta \sin n \theta$. Then for any given $\varepsilon>0$, there exists a set $H_{0} \subset[0,2 \pi)$ with linear measure zero, such that for any $\theta \in[0,2 \pi) \backslash\left(H_{0} \cup H_{1}\right)$, there exists $r_{0}=r_{0}(\theta, \varepsilon)(>0)$ such that for $|z|=r>r_{0}$, we have

(i) if $\delta(P, \theta)>0$, then

$$
\exp \left\{(1-\varepsilon) \delta(P, \theta) r^{n}\right\}<\left|g\left(r e^{i \theta}\right)\right|<\exp \left\{(1+\varepsilon) \delta(P, \theta) r^{n}\right\} ;
$$

(ii) if $\delta(P, \theta)<0$, then

$$
\exp \left\{(1+\varepsilon) \delta(P, \theta) r^{n}\right\}<\left|g\left(r e^{i \theta}\right)\right|<\exp \left\{(1-\varepsilon) \delta(P, \theta) r^{n}\right\},
$$

where $H_{1}=\{\theta \in[0,2 \pi): \delta(P, \theta)=0\}$ is a finite set.

Remark 2.2 Let $P(z)=a z^{n}+\cdots$ be a polynomial with degree $n$ and $z=r e^{i \theta}$, we denote $\delta(P, \theta)=|a| \cos (\arg a+n \theta)$.

Lemma 2.4 (see [17]) Let $f(z)$ be a nonconstant meromorphic function, $\varepsilon>0$, and $c \in$ $\mathbb{C} \backslash\{0\}$. If $\zeta=\sigma_{2}(f)<1$, then

$$
m\left(r, \frac{f(z+c)}{f(z)}\right)=o\left(\frac{T(r, f)}{r^{1-\zeta-\varepsilon}}\right)
$$

for all $r$ outside of a set of finite logarithmic measure.

Lemma 2.5 Let $c_{j}, j=1, \ldots, k$ be distinct nonzero complex constants, $A_{j}(z), j=0,1, \ldots, k$, $F(z)$ be meromorphic functions such that $A_{k}(z) A_{0}(z) F(z) \not \equiv 0$. If $f(z)$ is a meromorphic solution of the difference equation

$$
A_{k}(z) f\left(z+c_{k}\right)+\cdots+A_{1}(z) f\left(z+c_{1}\right)+A_{0}(z) f(z)=F(z)
$$

and satisfies $\max \left\{\sigma(F), \sigma\left(A_{j}\right), j=0,1, \ldots, k\right\}=\omega<\sigma(f)=\sigma(0<\sigma \leq \infty), \sigma_{2}(f)<1$, then we have

$$
\lambda(f)=\sigma(f) .
$$

Proof We use a similar proof to the one in [12] here. We rewrite (2.1) as

$$
\frac{1}{f(z)}=\frac{1}{F(z)}\left(A_{k}(z) \frac{f\left(z+c_{k}\right)}{f(z)}+\cdots+A_{1}(z) \frac{f\left(z+c_{1}\right)}{f(z)}+A_{0}(z)\right) .
$$

By Lemma 2.4, there exists a set $E \subset(1,+\infty)$ of finite logarithmic measure such that for all $z$ satisfying $|z|=r \notin E$, we have

$$
m\left(r, \frac{f\left(z+c_{j}\right)}{f(z)}\right)=o(T(r, f)), \quad j=1, \ldots, k .
$$


Thus, (2.2) implies that

$$
\begin{aligned}
T(r, f) & =N\left(r, \frac{1}{f}\right)+m\left(r, \frac{1}{f}\right)+O(1) \\
& \leq N\left(r, \frac{1}{f}\right)+m\left(r, \frac{1}{F}\right)+\sum_{j=0}^{k} m\left(r, A_{j}\right)+\sum_{j=1}^{k} m\left(r, \frac{f\left(z+c_{j}\right)}{f(z)}\right)+O(1) \\
& \leq N\left(r, \frac{1}{f}\right)+T(r, F)+\sum_{j=0}^{k} T\left(r, A_{j}\right)+o(T(r, f)), \quad r \notin E .
\end{aligned}
$$

Set $m_{l} E=\log \delta<\infty$. Since $\sigma(f)=\sigma$, there exists a sequence $\left\{r_{n}^{\prime}\right\}_{n=1}^{\infty}$ tending to $\infty$ such that $(\delta+2) r_{n}^{\prime}<r_{n+1}^{\prime}$ and

$$
\lim _{r_{n}^{\prime} \rightarrow \infty} \frac{\log T\left(r_{n}^{\prime}, f\right)}{\log r_{n}^{\prime}}=\sigma .
$$

We may choose $r_{n} \in\left[r_{n}^{\prime},(\delta+2) r_{n}^{\prime}\right] \backslash E, n=1,2, \ldots$. Since

$$
\frac{\log T\left(r_{n}, f\right)}{\log r_{n}} \geq \frac{\log T\left(r_{n}^{\prime}, f\right)}{\log (\delta+2) r_{n}^{\prime}}=\frac{\log T\left(r_{n}^{\prime}, f\right)}{\log r_{n}^{\prime}\left(1+\frac{\log (\delta+2)}{\log r_{n}^{\prime}}\right)}
$$

we have

$$
\underbrace{}_{r_{n} \rightarrow \infty} \frac{\log T\left(r_{n}, f\right)}{\log r_{n}} \geq \lim _{r_{n}^{\prime} \rightarrow \infty} \frac{\log T\left(r_{n}^{\prime}, f\right)}{\log r_{n}^{\prime}\left(1+\frac{\log (\delta+2)}{\log r_{n}^{\prime}}\right)}=\sigma
$$

that is

$$
\lim _{r_{n} \rightarrow \infty} \frac{\log T\left(r_{n}, f\right)}{\log r_{n}}=\sigma .
$$

Then for sufficiently small $\varepsilon\left(0<\varepsilon<\frac{\sigma-\omega}{2}\right)$ and sufficiently large $r_{n}$, we have

$$
T\left(r_{n}, f\right) \geq r_{n}^{\sigma-\varepsilon}
$$

and

$$
T\left(r_{n}, F\right) \leq r_{n}^{\omega+\varepsilon}, \quad T\left(r_{n}, A_{j}\right) \leq r_{n}^{\omega+\varepsilon}, \quad j=0,1, \ldots, k
$$

Hence,

$$
\frac{T\left(r_{n}, F\right)}{T\left(r_{n}, f\right)} \leq r_{n}^{\omega-\sigma+2 \varepsilon} \rightarrow 0, \quad r_{n} \rightarrow \infty
$$

and

$$
\frac{T\left(r_{n}, A_{j}\right)}{T\left(r_{n}, f\right)} \leq r_{n}^{\omega-\sigma+2 \varepsilon} \rightarrow 0, \quad r_{n} \rightarrow \infty, j=0,1, \ldots, k
$$


hold. Then, for sufficiently large $r_{n}$, we have

$$
\begin{aligned}
& T\left(r_{n}, F\right) \leq \frac{1}{k+4} T\left(r_{n}, f\right), \quad o\left(T\left(r_{n}, f\right)\right) \leq \frac{1}{k+4} T\left(r_{n}, f\right), \\
& T\left(r_{n}, A_{j}\right) \leq \frac{1}{k+4} T\left(r_{n}, f\right), \quad j=0,1, \ldots, k .
\end{aligned}
$$

It follows from (2.3) that for sufficiently large $r_{n}$,

$$
T\left(r_{n}, f\right) \leq N\left(r_{n}, \frac{1}{f}\right)+\frac{k+3}{k+4} T\left(r_{n}, f\right)
$$

that is,

$$
T\left(r_{n}, f\right) \leq(k+4) N\left(r_{n}, \frac{1}{f}\right)
$$

Therefore,

$$
\sigma=\lim _{r_{n} \rightarrow \infty} \frac{\log T\left(r_{n}, f\right)}{\log r_{n}} \leq \varlimsup_{r_{n} \rightarrow \infty} \frac{\log N\left(r_{n}, \frac{1}{f}\right)}{\log r_{n}} \leq \lambda(f),
$$

that is, $\lambda(f)=\sigma(f)$.

Remark 2.3 Noting that

$$
m\left(r, \frac{\Delta^{i} f}{f}\right) \leq O\left(\sum_{j=0}^{i} m\left(r, \frac{f(z+j c)}{f(z)}\right)\right), \quad i \in \mathbb{N}_{+}
$$

we see that if $f\left(z+c_{i}\right), i=1, \ldots, k$, in Lemma 2.5 are replaced, respectively, by $\Delta^{i} f, i=$ $1, \ldots, k$, the corresponding result holds.

\section{Proofs of Theorems 1.1 and 1.2}

Proof of Theorem 1.1 Suppose that $f(z)(\not \equiv 0)$ is a meromorphic solution of (1.1). If $\sigma(f)=$ $\infty$, then $\sigma(f) \geq n+1$ holds obviously. Now, we suppose $\sigma(f)=\sigma<\infty$. Set

$$
\begin{aligned}
& I_{1}=\left\{j \neq i \mid \arg a_{i n} \neq \arg a_{j n} \text { and } \arg a_{i n} \neq \arg b_{j n}\right\}, \\
& I_{2}=\left\{j \neq i \mid \arg a_{i n}=\arg b_{j n} \neq \arg a_{j n} \text { and }\left|a_{i n}\right|>\left|b_{j n}\right|\right\}, \\
& I_{3}=\left\{j \neq i \mid \arg a_{i n}=\arg a_{j n} \neq \arg b_{j n} \text { and }\left|a_{i n}\right|>\left|a_{j n}\right|\right\}, \\
& I_{4}=\left\{j \neq i \mid \arg a_{i n}=\arg b_{j n}=\arg a_{j n} \text { and }\left|a_{i n}\right|>\left|a_{j n}\right| \geq\left|b_{j n}\right|\right\} .
\end{aligned}
$$

It is clear that $I_{1}, I_{2}, I_{3}, I_{4}$ do not intersect with each other, and $I=I_{1} \cup I_{2} \cup I_{3} \cup I_{4}=$ $\{0,1, \ldots, k\} \backslash\{i\}$.

Now, we may choose $\theta_{0} \in(0,2 \pi)$ such that $\cos \left(\arg a_{i n}+n \theta_{0}\right)=1$. (If $n=1$ and $\arg a_{i n}=0$, then we replace $[0,2 \pi)$ in Lemma 2.3 by $\left[-\frac{\pi}{2}, \frac{3 \pi}{2}\right)$; if $n \geq 2$, this kind of $\theta_{0}$ can always be chosen.) Set $z=r e^{i \theta}, \theta \in[0,2 \pi)$. 
For $j \in I_{1}$, there exists sufficiently small $\varepsilon_{1}(>0)$ such that for all $\theta \in\left(\theta_{0}-\varepsilon_{1}, \theta_{0}+\varepsilon_{1}\right) \subset$ $(0,2 \pi)$, we have

$$
\cos \left(\arg a_{i n}+n \theta\right)>\max _{j \in I_{1}}\left\{\cos \left(\arg a_{j n}+n \theta\right), \cos \left(\arg b_{j n}+n \theta\right), 0\right\}
$$

Since $\left|a_{i n}\right| \geq\left|a_{j n}\right| \geq\left|b_{j n}\right|>0, j \neq i$, we see that

$$
\delta\left(P_{i}, \theta\right)>\max _{j \in I_{1}}\left\{\delta\left(P_{j}, \theta\right), \delta\left(Q_{j}, \theta\right), 0\right\}
$$

For $j \in I_{2}$, there exists sufficiently small $\varepsilon_{2}(>0)$ such that for all $\theta \in\left(\theta_{0}-\varepsilon_{2}, \theta_{0}+\varepsilon_{2}\right) \subset$ $(0,2 \pi)$, we have

$$
\cos \left(\arg a_{i n}+n \theta\right)=\cos \left(\arg b_{j n}+n \theta\right)>\max _{j \in I_{2}}\left\{\cos \left(\arg a_{j n}+n \theta\right), 0\right\} .
$$

Since $\left|a_{i n}\right|>\left|b_{j n}\right|, j \neq i$, and $\left|a_{i n}\right| \geq\left|a_{j n}\right|$, we see that

$$
\delta\left(P_{i}, \theta\right)>\max _{j \in I_{2}}\left\{\delta\left(P_{j}, \theta\right), \delta\left(Q_{j}, \theta\right)\right\}>0
$$

For $j \in I_{3}$, there exists sufficiently small $\varepsilon_{3}(>0)$ such that for all $\theta \in\left(\theta_{0}-\varepsilon_{3}, \theta_{0}+\varepsilon_{3}\right) \subset$ $(0,2 \pi)$, we have

$$
\cos \left(\arg a_{i n}+n \theta\right)=\cos \left(\arg a_{j n}+n \theta\right)>\max _{j \in I_{3}}\left\{\cos \left(\arg b_{j n}+n \theta\right), 0\right\} .
$$

Since $\left|a_{i n}\right|>\left|a_{j n}\right| \geq\left|b_{j n}\right|, j \neq i$, we see that

$$
\delta\left(P_{i}, \theta\right)>\max _{j \in I_{3}}\left\{\delta\left(P_{j}, \theta\right), \delta\left(Q_{j}, \theta\right)\right\}>0 .
$$

For $j \in I_{4}$, there exists sufficiently small $\varepsilon_{4}(>0)$ such that for all $\theta \in\left(\theta_{0}-\varepsilon_{4}, \theta_{0}+\varepsilon_{4}\right) \subset$ $(0,2 \pi)$, we have

$$
\cos \left(\arg a_{i n}+n \theta\right)=\cos \left(\arg b_{j n}+n \theta\right)=\cos \left(\arg a_{j n}+n \theta\right)>0 .
$$

Since $\left|a_{i n}\right|>\left|a_{j n}\right| \geq\left|b_{j n}\right|$, we see that

$$
\delta\left(P_{i}, \theta\right)>\max _{j \in I_{4}}\left\{\delta\left(P_{j}, \theta\right), \delta\left(Q_{j}, \theta\right)\right\}>0
$$

Set $\varepsilon_{0}^{\prime}=\min \left\{\varepsilon_{1}, \varepsilon_{2}, \varepsilon_{3}, \varepsilon_{4}\right\}$, then for any $\theta \in\left(\theta_{0}-\varepsilon_{0}^{\prime}, \theta_{0}+\varepsilon_{0}^{\prime}\right) \subset(0,2 \pi)$, we have

$$
\delta\left(P_{i}, \theta\right)=\delta_{1}>\max _{j \in I}\left\{\delta\left(P_{j}, \theta\right), \delta\left(Q_{j}, \theta\right), 0\right\}=\delta_{2}
$$

If $\left|b_{i n}\right|<\left|a_{i n}\right|$ and $\arg b_{i n}=\arg a_{i n}$, then for all $\theta \in\left(\theta_{0}-\varepsilon_{0}^{\prime}, \theta_{0}+\varepsilon_{0}^{\prime}\right) \subset(0,2 \pi)$, we have

$$
\delta\left(P_{i}, \theta\right)>\delta\left(Q_{i}, \theta\right)>0
$$


If $\arg a_{i n} \neq \arg b_{\text {in }}$ and $\left|b_{i n}\right| \leq\left|a_{i n}\right|$, then there exists $\varepsilon_{0}\left(0<\varepsilon_{0} \leq \varepsilon_{0}^{\prime}\right)$ such that for all $\theta \in$ $\left(\theta_{0}-\varepsilon_{0}, \theta_{0}+\varepsilon_{0}\right) \subset(0,2 \pi)$, we have

$$
\cos \left(\arg a_{i n}+n \theta\right)>\max \left\{\cos \left(\arg b_{i n}+n \theta\right), 0\right\}
$$

and, correspondingly,

$$
\delta\left(P_{i}, \theta\right)>\max \left\{\delta\left(Q_{i}, \theta\right), 0\right\} .
$$

Therefore, if $b_{\text {in }} \neq a_{i n}$, and $\left|b_{\text {in }}\right| \leq\left|a_{i n}\right|$, then there exists $\varepsilon_{0}(>0)$ such that for all $\theta \in\left(\theta_{0}-\right.$ $\left.\varepsilon_{0}, \theta_{0}+\varepsilon_{0}\right) \subset(0,2 \pi)$, we have

$$
\delta\left(P_{i}, \theta\right)=\delta_{1}>\max \left\{\delta\left(Q_{i}, \theta\right), 0\right\}=\delta_{3} .
$$

If $b_{\text {in }}=a_{i n}$, then $A_{i}(z)=\tilde{B}_{i}(z) e^{a_{i n} z^{n}}+R_{i}(z)$, where $\tilde{B}_{i}(z)$ is a meromorphic function and satisfies $\sigma\left(\tilde{B}_{i}\right) \leq \max \{\omega, n-1\}$. Since $A_{i}(z)-R_{i}(z) \not \equiv 0$, we see that $\tilde{B}_{i}(z) \not \equiv 0$. Here, $D_{i}(z) \equiv 0$, and

$$
\delta\left(a_{i n} z^{n}, \theta\right)=\delta\left(P_{i}, \theta\right)=\delta_{1}>0 .
$$

By Lemma 2.2, for any given $\varepsilon\left(0<\varepsilon<\frac{1}{2} \min \left\{\frac{\delta_{1}-\delta_{2}}{\delta_{1}+\delta_{2}}, \frac{\delta_{1}-\delta_{3}}{\delta_{1}+\delta_{3}}, n-\omega\right\}\right)$, there exists a set $E_{1} \subset$ $(0,+\infty)$ with finite linear measure, such that for all $z$ satisfying $|z|=r \notin E_{1}, j=0,1, \ldots, k$ and $r \rightarrow \infty$, we have

$$
\left|B_{j}(z)\right| \leq \exp \left\{r^{\omega+\varepsilon}\right\}, \quad\left|D_{j}(z)\right| \leq \exp \left\{r^{\omega+\varepsilon}\right\}, \quad\left|R_{j}(z)\right| \leq \exp \left\{r^{\omega+\varepsilon}\right\} .
$$

By Lemma 2.3 and (3.1)-(3.4), for the above $\varepsilon>0$, there exists $H_{0} \subset[0,2 \pi)$ with linear measure zero and a finite set $H_{1}=\bigcup_{i=0}^{k}\left\{\theta \in[0,2 \pi) \mid \delta\left(P_{i}, \theta\right)=0\right.$ or $\left.\delta\left(Q_{i}, \theta\right)=0\right\}$, such that for all $\theta \in\left(\theta_{0}-\varepsilon_{0}, \theta_{0}+\varepsilon_{0}\right) \backslash\left(H_{0} \cup H_{1}\right)$, there exists $r_{0}=r_{0}(\theta, \varepsilon)>0$, such that for $r>r_{0}$, we have

$$
\begin{aligned}
\left|A_{j}(z)\right| & \leq\left|B_{j}(z) e^{P_{j}(z)}\right|+\left|D_{j}(z) e^{Q_{j}(z)}\right|+\left|R_{j}(z)\right| \\
& \leq 2 \exp \left\{(1+\varepsilon) \delta_{2} r^{n}\right\}+\exp \left\{r^{\omega+\varepsilon}\right\} \\
& \leq 3 \exp \left\{(1+\varepsilon) \delta_{2} r^{n}\right\} \exp \left\{r^{\omega+\varepsilon}\right\}, \quad j \neq i
\end{aligned}
$$

and

$$
\begin{aligned}
\left|A_{i}(z)\right| & \geq\left|B_{i}(z) e^{P_{i}(z)}\right|-\left|D_{i}(z) e^{Q_{i}(z)}\right|-\left|R_{i}(z)\right| \\
& \geq \exp \left\{(1-\varepsilon) \delta_{1} r^{n}\right\}-\exp \left\{(1+\varepsilon) \delta_{3} r^{n}\right\}-\exp \left\{r^{\omega+\varepsilon}\right\} \\
& \geq \exp \left\{(1-\varepsilon) \delta_{1} r^{n}\right\}-2 \exp \left\{(1+\varepsilon) \delta_{3} r^{n}\right\} \exp \left\{r^{\omega+\varepsilon}\right\} .
\end{aligned}
$$

By Lemma 2.1, for the above $\varepsilon>0$, there exists a set $E_{2} \subset(1,+\infty)$ with finite logarithmic measure, such that for all $z$ satisfying $|z|=r \notin E_{2} \cup[0,1]$, we have

$$
\left|\frac{f\left(z+c_{j}\right)}{f\left(z+c_{i}\right)}\right| \leq \exp \left\{r^{\sigma-1+\varepsilon}\right\}, \quad j \neq i .
$$


Equation (1.1) gives

$$
\begin{aligned}
-A_{i}(z)= & A_{k}(z) \frac{f\left(z+c_{k}\right)}{f\left(z+c_{i}\right)}+\cdots+A_{i+1}(z) \frac{f\left(z+c_{i+1}\right)}{f\left(z+c_{i}\right)} \\
& +A_{i-1}(z) \frac{f\left(z+c_{i-1}\right)}{f\left(z+c_{i}\right)}+\cdots+A_{0}(z) \frac{f(z)}{f\left(z+c_{i}\right)}
\end{aligned}
$$

and, correspondingly,

$$
\begin{aligned}
\left|A_{i}(z)\right| \leq & \left|A_{k}(z)\right|\left|\frac{f\left(z+c_{k}\right)}{f\left(z+c_{i}\right)}\right|+\cdots+\left|A_{i+1}(z)\right|\left|\frac{f\left(z+c_{i+1}\right)}{f\left(z+c_{i}\right)}\right| \\
& +\left|A_{i-1}(z)\right|\left|\frac{f\left(z+c_{i-1}\right)}{f\left(z+c_{i}\right)}\right|+\cdots+\left|A_{0}(z)\right| \frac{f(z)}{f\left(z+c_{i}\right)} \mid .
\end{aligned}
$$

It follows from (3.5)-(3.8) that for all $z=r e^{i \theta}$, where $\theta \in\left(\theta_{0}-\varepsilon_{0}, \theta_{0}+\varepsilon_{0}\right) \backslash\left(H_{0} \cup H_{1}\right), r \notin$ $[0,1] \cup E_{1} \cup E_{2}$ and $r \rightarrow \infty$, we have

$$
\begin{array}{r}
\exp \left\{(1-\varepsilon) \delta_{1} r^{\eta}\right\}-2 \exp \left\{(1+\varepsilon) \delta_{3} r^{n}\right\} \exp \left\{r^{\omega+\varepsilon}\right\} \\
\leq 3 k \exp \left\{r^{\sigma-1+\varepsilon}\right\} \exp \left\{(1+\varepsilon) \delta_{2} r^{\eta}\right\} \exp \left\{r^{\omega+\varepsilon}\right\} .
\end{array}
$$

Since $0<\varepsilon<\frac{1}{2} \min \left\{\frac{\delta_{1}-\delta_{2}}{\delta_{1}+\delta_{2}}, \frac{\delta_{1}-\delta_{3}}{\delta_{1}+\delta_{3}}, n-\omega\right\}$, we have

$$
(1+\varepsilon) \delta_{2}<(1-2 \varepsilon) \delta_{1}, \quad(1+\varepsilon) \delta_{3}<(1-2 \varepsilon) \delta_{1}, \quad \omega+\varepsilon<n-\varepsilon
$$

and

$$
\frac{2 \exp \left\{(1+\varepsilon) \delta_{3} r^{n}\right\} \exp \left\{r^{\omega+\varepsilon}\right\}}{\exp \left\{(1-\varepsilon) \delta_{1} r^{n}\right\}}<2 \exp \left\{r^{\omega+\varepsilon}-\varepsilon \delta_{1} r^{n}\right\} \rightarrow 0, \quad r \notin[0,1] \cup E_{1} \cup E_{2}, r \rightarrow \infty .
$$

Then from (3.9), for sufficiently large $r$, we have

$$
\frac{1}{2} \exp \left\{(1-\varepsilon) \delta_{1} r^{n}\right\} \leq 3 k \exp \left\{r^{\sigma-1+\varepsilon}\right\} \exp \left\{(1+\varepsilon) \delta_{2} r^{n}\right\} \exp \left\{r^{\omega+\varepsilon}\right\}
$$

i.e.,

$$
\exp \left\{(1-\varepsilon) \delta_{1} r^{n}-(1+\varepsilon) \delta_{2} r^{n}-r^{\omega+\varepsilon}\right\} \leq 6 k \exp \left\{r^{\sigma-1+\varepsilon}\right\}
$$

i.e.,

$$
\exp \left\{\frac{1}{2} \varepsilon \delta_{1} r^{n}\right\} \leq 6 k \exp \left\{r^{\sigma-1+\varepsilon}\right\}
$$

Then (3.10) implies $n \leq \sigma-1+\varepsilon$. Since $\varepsilon$ is arbitrary, we have $\sigma(f)=\sigma \geq n+1$.

Therefore, every meromorphic solution $f(z)(\not \equiv 0)$ satisfies $\sigma(f) \geq n+1$.

Set $g(z)=f(z)-\varphi(z)$, then $g(z)$ solves the equation

$$
\begin{aligned}
& A_{k}(z) g\left(z+c_{k}\right)+\cdots+A_{1}(z) g\left(z+c_{1}\right)+A_{0}(z) g(z) \\
& \quad=-A_{k}(z) \varphi\left(z+c_{k}\right)-\cdots-A_{1}(z) \varphi\left(z+c_{1}\right)-A_{0}(z) \varphi(z) .
\end{aligned}
$$


Since $\sigma(\varphi)<n+1, \varphi(z)(\not \equiv 0)$ does not solve (1.1), that is,

$$
A_{k}(z) \varphi\left(z+c_{k}\right)+\cdots+A_{1}(z) \varphi\left(z+c_{1}\right)+A_{0}(z) \varphi(z) \not \equiv 0
$$

and

$$
\begin{aligned}
& \sigma\left(A_{k}(z) \varphi\left(z+c_{k}\right)+\cdots+A_{1}(z) \varphi\left(z+c_{1}\right)+A_{0}(z) \varphi(z)\right) \\
& \quad \leq \max \left\{\sigma\left(A_{j}\right), j=0,1, \ldots, k, \sigma(\varphi)\right\} \\
& \quad<n+1 \leq \sigma(f) .
\end{aligned}
$$

Therefore, by Lemma 2.5, we have $\lambda(g)=\sigma(g)$, i.e., $\lambda(f-\varphi)=\sigma(f)$.

The proof of Theorem 1.1 is completed.

Proof of Theorem 1.2 Equation (1.2) gives

$$
-A_{0}(z)=A_{k}(z) \frac{\Delta^{k} f(z)}{f(z)}+\cdots+A_{1}(z) \frac{\Delta f(z)}{f(z)} .
$$

By Remark 2.1, we have

$$
\left|\frac{\Delta^{j} f(z)}{f(z)}\right| \leq O\left(\exp \left\{r^{\sigma-1+\varepsilon}\right\}\right), \quad j=1, \ldots, k
$$

By combining (3.5), (3.6), (3.12) with (3.11), for all $z=r e^{i \theta}$, where $\arg z=\theta \in\left(\theta_{0}-\varepsilon_{0}, \theta_{0}+\right.$ $\left.\varepsilon_{0}\right) \backslash\left(H_{0} \cup H_{1}\right),|z|=r \notin[0,1] \cup E_{1} \cup E_{2}$ and for sufficiently large $r$, we have

$$
\begin{aligned}
& \exp \left\{(1-\varepsilon) \delta_{1} r^{n}\right\}-2 \exp \left\{(1+\varepsilon) \delta_{3} r^{n}\right\} \exp \left\{r^{\omega+\varepsilon}\right\} \\
& \leq O\left(\exp \left\{r^{\sigma-1+\varepsilon}\right\}\right) \exp \left\{(1+\varepsilon) \delta_{2} r^{n}\right\} \exp \left\{r^{\omega+\varepsilon}\right\} .
\end{aligned}
$$

By using a similar method to the one in the proof of Theorem 1.1, we have $\sigma(f)=\sigma \geq n+1$.

Further, set $g(z)=f(z)-\varphi(z)$, then $g(z)$ solves the equation

$$
\begin{aligned}
& A_{k}(z) \Delta^{k} g(z)+\cdots+A_{1}(z) \Delta g(z)+A_{0}(z) g(z) \\
& \quad=-A_{k}(z) \Delta^{k} \varphi(z)-\cdots-A_{1}(z) \Delta \varphi(z)-A_{0}(z) \varphi(z) .
\end{aligned}
$$

Since $\sigma(\varphi)<n+1, \varphi(z)(\not \equiv 0)$ does not solve (1.2), that is,

$$
A_{k}(z) \Delta^{k} \varphi(z)+\cdots+A_{1}(z) \Delta \varphi(z)+A_{0}(z) \varphi(z) \not \equiv 0
$$

and

$$
\sigma\left(A_{k} \Delta^{k} \varphi+\cdots+A_{1} \Delta \varphi+A_{0} \varphi\right) \leq \max \left\{\sigma\left(A_{j}\right), j=0,1, \ldots, k, \sigma(\varphi)\right\}<n+1 \leq \sigma(f) .
$$

Therefore, by Lemma 2.5 and Remark 2.3, we have $\lambda(g)=\sigma(g)$, i.e., $\lambda(f-\varphi)=\sigma(f)$.

The proof of Theorem 1.2 is completed. 
Competing interests

The authors declare that they have no competing interests.

\section{Authors' contributions}

All authors drafted the manuscript, and they read and approved the final manuscript.

\section{Acknowledgements}

This work was supported by the National Natural Science Foundation of China $(11301233,11171119)$, the Natural Science Foundation of Jiangxi Province in China (20151BAB201004), and the Youth Science Foundation of Education Bureau of Jiangxi Province in China (GJJ14271).

Received: 2 February 2015 Accepted: 24 April 2015 Published online: 07 May 2015

\section{References}

1. Hayman, WK: Meromorphic Functions. Clarendon, Oxford (1964)

2. Laine, I: Nevanlinna Theory and Complex Differential Equations. de Gruyter, Berlin (1993)

3. Yang, L: Value Distribution Theory and Its New Research. Science Press, Beijing (1982) (in Chinese)

4. Yi, HX, Yang, CC: Uniqueness Theory of Meromorphic Functions. Mathematics and Its Applications, vol. 557. Kluwer Academic, Dordrecht (2003)

5. Chen, ZX, Shon, KH: On growth of meromorphic solutions for linear difference equations. Abstr. Appl. Anal. 2013, Article ID 619296 (2013)

6. Chiang, YK, Feng, SJ: On the Nevanlinna characteristic of $f(z+\eta)$ and difference equations in the complex plane. Ramanujan J. 16(1), 105-129 (2008)

7. Li, S, Gao, ZS: Finite order meromorphic solutions of linear difference equations. Proc. Jpn. Acad., Ser. A, Math. Sci. 87(5), 73-76 (2011)

8. Laine, I, Yang, CC: Clunie theorems for difference and q-difference polynomials. J. Lond. Math. Soc. 76(2), 556-566 (2007)

9. Latreuch, Z, Belaïdi, B: Growth and oscillation of meromorphic solutions of linear difference equations. Mat. Vesn. 66(2), 213-222 (2014)

10. Zheng, XM, Tu, J: Growth of meromorphic solutions of linear difference equations. J. Math. Anal. Appl. 384(2), 349-356 (2011)

11. Chen, ZX: Zeros of entire solutions to complex linear difference equations. Acta Math. Sci., Ser. B 32(3), 1141-1148 (2012)

12. Liu, YX: On growth of meromorphic solutions for linear difference equations with meromorphic coefficients. Adv. Differ. Equ. 2013, 60 (2013)

13. Liu, HF, Mao, ZQ: On the meromorphic solutions of some linear difference equations. Adv. Differ. Equ. 2013, 133 (2013)

14. Bergweiler, W, Langley, JK: Zeros of differences of meromorphic functions. Math. Proc. Camb. Philos. Soc. 142(1), 133-147 (2007)

15. Chen, ZX: The growth of solutions of a type of second order differential equations with entire coefficients. Chin. Ann. Math., Ser. A 20(1), 7-14 (1999) (in Chinese)

16. $\mathrm{Cao}, \mathrm{CL}$, Chen, $\mathrm{ZX}$ : On the orders and zeros of the solutions of certain linear differential equations with entire coefficients. Acta Math. Appl. Sin. 25(1), 123-131 (2002) (in Chinese)

17. Halburd, RG, Korhonen, RJ, Toghe, K: Holomorphic curves with shift-invariant hyperplane preimages. Trans. Am. Math. Soc. 336(8), 4267-4298 (2014)

\section{Submit your manuscript to a SpringerOpen ${ }^{\odot}$ journal and benefit from:}

- Convenient online submission

Rigorous peer review

- Immediate publication on acceptance

- Open access: articles freely available online

- High visibility within the field

- Retaining the copyright to your article 